\title{
BMJ Open Predictive model for patients with poor-grade subarachnoid haemorrhage in 30-day observation: a 9-year cohort study
}

\author{
Sebastian Szklener, ${ }^{1}$ Anna Melges, ${ }^{1}$ Agnieszka Korchut, ${ }^{1}$ Wojciech Zaluska, ${ }^{2}$ \\ Tomasz Trojanowski, ${ }^{3}$ Robert Rejdak, ${ }^{4}$ Konrad Rejdak ${ }^{1}$
}

To cite: Szklener S, Melges A, Korchut A, et al. Predictive model for patients with poor-grade subarachnoid haemorrhage in 30-day observation: a 9-year cohort study. BMJ Open 2015;5:e007795. doi:10.1136/bmjopen-2015007795

- Prepublication history for this paper is available online. To view these files please visit the journal online (http://dx.doi.org/10.1136/ bmjopen-2015-007795).

Received 27 January 2015 Revised 19 February 2015 Accepted 20 March 2015

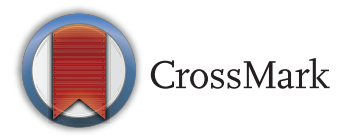

${ }^{1}$ Department of Neurology, Medical University of Lublin, Poland

${ }^{2}$ Department of Nephrology, Medical University of Lublin, Poland

${ }^{3}$ Department of

Neurosurgery, Medical University of Lublin, Poland

${ }^{4}$ Department of General Ophthalmology, Medical University of Lublin, Poland

Correspondence to Dr Sebastian Szklener; szklener@wp.pl

\section{ABSTRACT}

Objective: The purpose of this study was to identify prognostic factors and build the predictive model based on poor-grade subarachnoid haemorrhage (SAH) population received only supportive symptomatic treatment.

Design: Prospective observational cohort study. Setting: Intensive care unit at the Clinical Department of Neurology.

Participants: A total of 101 patients with spontaneous SAH disqualified from neurosurgical operative treatment due to poor clinical condition. Data were collected over a 9-year period.

Outcome measures: Unfavourable outcome was defined as a modified Rankin Score $\geq 5$ at 30 days of observation.

Results: Multivariable logistic regression analysis indicated the World Federation of Neurosurgical Societies Scale score, increasing age, Fisher grade and admission leucocytosis as independent predictive factors. The proposed scale subdivides the study population into four prognostic groups with significantly different outcomes: grade I: probability of favourable outcome $89.9 \%$; grade II: $47.5 \%$; grade III: $4.2 \%$; grade IV: $0 \%$. The receiver operating characteristic (ROC) curve for the prediction of outcome performed by the new scale had an area under the curve (AUC) $=0.910$ (excellent accuracy).

Conclusions: Unfavourable outcome in non-operated patients with poor-grade SAH is strongly predicted by traditional unmodifiable factors such as age, amount of bleeding in CT, level of consciousness as well as leucocytosis. A new predictive scale based on the above parameters seems to reliably predict the outcome and may contribute to more effective planning of therapeutic management in patients with poor-grade SAH.

\section{INTRODUCTION}

Bleeding into the subarachnoid space (subarachnoid haemorrhage, SAH) is a serious medical condition with complex pathogenesis and variable clinical presentation. Surgery or

\section{Strengths and limitations of this study}

- There is a lack of studies analysing the population of patients with poor-grade $\mathrm{SAH}$, which indeed represent a special cohort of patients, particularly due to the different clinical course with extremely high mortality and morbidity rates as well as the undefined clear treatment protocol.

- The proposed model provides an opportunity for easy use and early outcome prediction among patients with SAH in poor clinical condition which may contribute to more effective planning of therapeutic management.

- One potentially uncontrolled confounder is appropriate evaluation of morphological parameter of aneurysm.

endovascular treatment is a key component for the proper management of patients with SAH. However, the implementation of definitive therapy for patients in poor-grade $\mathrm{SAH}$ remains controversial due to its association with the high morbidity and mortality rates. ${ }^{12}$ These patients are usually managed conservatively and those who survive and show clinical improvement are selected for definitive therapy. In recent years, there have been several studies indicating an improved outcome with the early treatment of the ruptured aneurysm in a selected subgroup of patients with the poor-grade aneurysmal SAH. ${ }^{1-3}$ Undoubtedly, early aneurysm occlusion prevents recurrent haemorrhage and vasospasm can be treated more effectively, yet nothing can be done about irreversible brain damage. Moreover, severe cerebral insult is not directly expressed in neurological condition at admission. Clinical presentation of poor-grade $\mathrm{SAH}$ is a highly complex process caused by intracerebral haemorrhage, cerebral swelling, intraventricular haemorrhage, acute hydrocephalus, microcirculatory disturbances, decreased global cerebral perfusion, 
increased intracranial pressure and neurogenic cardiac or pulmonary dysfunction. ${ }^{4}$ Therefore, a more comprehensive evaluation of clinical condition is essential for the proper outcome prediction, especially in regard to this cohort. There has been an enormous effort to work out the prognostic models predicting an outcome in patients with SAH over many years. ${ }^{5}$ This was largely complicated by heterogeneity of study populations and treatment procedures as well as approaches having been used, all of which influenced the outcome. Those studies included different subgroups of patients exposed to various treatment procedures with the majority of patients in good-grade SAH who qualified for surgical treatment. Only a few patients in these cohorts were not operated on and they were exposed only to supportive symptomatic treatment. Indeed, patients with poor-grade $\mathrm{SAH}$, who received no surgical treatment, represent a special population that deserves particular attention, especially from the view of neurological wards.

In this study, we focused on such a non-operated, poorgrade $\mathrm{SAH}$ population, treated in a neurological intensive care unit (NICU), so as to identify predictive factors and build the prognostic model for such a population.

\section{MATERIALS AND METHODS}

The study was conducted on a group of patients who had been admitted and treated with diagnosed spontaneous SAH (ie, bleeding into subarachnoid space confirmed by a CT scan). The research protocol was planned for the prospective observational clinical study carried out in the period from January 2001 to December 2010. After completion of the data collection, the retrospective analysis was performed.

The study group only included patients disqualified from neurosurgical clipping or endovascular coiling due to poor clinical condition (ie, World Federation of Neurological Surgeons Grades IV and V).

Exclusion criteria included: (1) patients aged $<18$ years; (2) admission criteria $>24 \mathrm{~h}$; (3) perimesencephalic patterns of haemorrhage on CT scans; (4) patients who were admitted in poor grades to the NICU but after initial treatment were qualified for operative treatmentonly patients managed conservatively during the observation period were included in the analysis; (5) posttraumatic haemorrhage, intoxication and the presence of any other serious medical conditions (active cancer, infectious, systemic, haematological, cardiovascular diseases, etc) that had existed prior to the occurrence of $\mathrm{SAH}$ and could otherwise have led to the significant disruption of the correct assessment of the clinical status and outcome among patients with SAH.

The study was approved by the hospital's institutional review board.

\section{Collected data}

After admission, the patients were managed in accordance with a standardised protocol used in our hospital. We considered all patient characteristics that could be collected reliably within the first hours after hospital admission. The clinical status of the patients was evaluated by the World Federation of Neurological Surgeons Grading System for Subarachnoid Hemorrhage (WFNS). ${ }^{6}$ At the same time, blood samples were collected and basic laboratory tests were performed. On the basis of routinely collected data, potential predicting factors of unfavourable outcome were analysed according to strict definitions. They included: (1) age (age tertiles were rounded to the nearest decade of age: $\leq 50,51-65,>65$ years) and sex; (2) medical history (cardiac arrhythmia, hypertension, ischaemic heart disease, diabetes); (3) admission laboratory findings; leucocytosis (white cell count (WCC) $>15 \times 10^{9} / \mathrm{L}$ ), ${ }^{78}$ anaemia (haemoglobin $<9.0 \mathrm{~g} / \mathrm{L}$ ), hyperglycaemia (blood glucose $\geq 7.8 \mathrm{mmol} / \mathrm{L}$ ) ${ }^{9}$ hyponatraemia (serum sodium $\leq 130 \mathrm{mmol} / \mathrm{L}$ ); (4) clinical factors; fever (body temperature $\geq 38.0^{\circ} \mathrm{C}$ ), tachycardia (heart rate $>100 / \mathrm{min}$ ), bradycardia (heart rate $<60 / \mathrm{min}$ ), systolic blood pressure (categories: <130, 130-139, 140-159, 160-179, 180-209, $\geq 210 \mathrm{~mm} \mathrm{Hg}$ ) and diastolic blood pressure (categories: $<85$, 85-89, 90-99, 100-109, 110-119, $\geq 120$ mm Hg); (5) radiological variables (Fisher scale). ${ }^{10}$ The disease and its consequences were evaluated by means of a modified six-point Rankin scale (mRS) during a 30-day long hospital observation in the local clinic.

\section{STATISTICAL METHODS}

The univariate logistic regression was conducted on the basis of 17 factors, depending on the dichotomous outcome division of the study group (favourable and unfavourable). In view of the initial poor clinical condition, observation period and limited spread of outcome seen in poor-grade patients, favourable outcome was defined as an mRS score of $0-4$ and unfavourable outcome as mRS scores of 5 and 6 . Similar methods were followed in other studies in the population of patients with poor-grade SAH. ${ }^{11}$ Continuous variables were categorised to aid in a clinical application. Statistical analysis was allowed to determine five factors that are correlated with the prognosis. Thereafter, a multivariate logistic regression analysis was carried out using backward elimination to identify independent predictors $(p<0.05)$. The relative weighting of each individual component was determined from the relative change of the OR of the variables. On the basis of the collected data, a nine grades prognostic model was developed. Subsequently, the grading system was compressed to four grades for simplification of the tool. The sensitivity and specificity of the new scale was determined, and the result has allowed to determine the reliability of the diagnostic test by receiver operating characteristic (ROC) curve. In addition, to test the prediction value of the new scale in relation to mortality, the Kaplan-Meier survival curves were performed. Statistical analyses were performed using MedCalc V.12.2 (MedCalc Software, Ostend, Belgium) or Stata V.11 (StataCorp, College Station, Texas, USA). 


\section{RESULTS}

\section{Baseline demographic and clinical data}

A group of 101 of 231 patients (114 women and 117 men) fulfilled the inclusion criteria for the study. Demographic and clinical features classified by the outcome are shown in table 1 . The age of the patients ranged from 21 to 87 years with a mean age of 57 years, which presented a linear relationship with the outcome. Male-to-female ratio was 1.4:1 (58 men and 43 women). According to medical history, hypertension was present in half of the cases, whereas other cardiovascular diseases were less frequent (cardiac arrhythmia 9\%, coronary artery disease $6 \%$ and diabetes mellitus $7 \%$ ). However, no chronic disease that was analysed affected the outcome. Of a total number of poor-grade patients, 27 $(27 \%)$ were in WFNS grade IV and $74(73 \%)$ in grade V. Having assessed the CT scans on arrival of those patients, most patients $(94 \%)$ were in Fisher grade III $(31 \%)$ and IV (63\%). Angiogram (both digital subtraction angiography and/or CT angiogram) examination was attempted in all study subjects. In the total group of patients with SAH, the intracranial aneurysms were precisely identified in 27 cases, and the arteriovenous malformation was found in two patients, who, however, were not excluded from the study because the sample was too small to disrupt the final results. Among the rest of the patients, the direct source of bleeding was not exactly identified.

Out of those who were admitted to our department and subsequently analysed, $21(21 \%)$ were found in an improved clinical condition after 30 days of the SAH treatment. Unfortunately, 80 patients $(79 \%)$ had an unfavourable outcome, including in-hospital mortality that occurred in 74 cases $(73 \%)$. Out of these deaths, 52 were due to the initial haemorrhage, 14 were due to rebleeding documented by CT, 5 were due to vasospasm, 2 were due to medical complication and one was due to unknown causes.

\section{Outcome predictors}

The conducted multivariate analysis, which incorporates all five predictors that are relevant in simple models, has identified four independent predictors. The level of consciousness by WFNS score $(\mathrm{OR}=16.52 ; \mathrm{p}<0.0001)$, increasing age $(\mathrm{OR}=4.9 ; \mathrm{p}=0.0053)$, amount of bleeding in CT estimated by the Fisher scale $(\mathrm{OR}=4.23 ; \mathrm{p}=0.0051)$ and admission leucocytosis $(\mathrm{OR}=6.16 ; \mathrm{p}=0.0186)$ achieved the strongest predictive value. The Hosmer and Lemeshow test $\left(\chi^{2}\right.$ goodness-of-fit=3.0342; $\left.\mathrm{p}=0.9322\right)$ has indicated good calibration of the multivariate logistic model (table 2).

\begin{tabular}{|c|c|c|c|}
\hline \multirow[b]{2}{*}{ Variable } & \multicolumn{2}{|c|}{ Number of patients (\%) } & \multirow[b]{2}{*}{ Overall $p$ value } \\
\hline & Good outcome & Poor outcome & \\
\hline Total group & $21 / 101$ & $80 / 101$ & - \\
\hline Age, year & & & 0.005 \\
\hline$\leq 50$ & 13 & 21 & \\
\hline $51-65$ & 6 & 30 & \\
\hline$>65$ & 2 & 29 & \\
\hline Male/female & $16 / 5$ & $42 / 38$ & 0.0573 (NS) \\
\hline Hypertension & $8(38)$ & $44(55)$ & 0.172 (NS) \\
\hline Coronary artery disease & $1(5)$ & $5(6)$ & 0.789 (NS) \\
\hline Cardiac arrhythmia & $1(5)$ & $8(10)$ & 0.464 (NS) \\
\hline Diabetes mellitus & $2(10)$ & $5(7)$ & 0.6017 (NS) \\
\hline Hyperglycaemia ( $\geq 7.8 \mathrm{mmol} / \mathrm{L})$ & $8(52)$ & $50(68)$ & 0.0458 \\
\hline Hyponatraemia (<130 mmol/L) & $1(5)$ & $8(10)$ & 0.4406 (NS) \\
\hline Anaemia (haemoglobin $<9.0 \mathrm{~g} / \mathrm{L}$ ) & $1(5)$ & $1(1)$ & 0.3527 (NS) \\
\hline 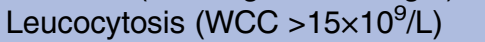 & $5(24)$ & $41(50)$ & 0.03 \\
\hline Systolic blood pressure (median) & 130-139 & $130-139$ & 0.4491 (NS) \\
\hline Diastolic blood pressure (median) & $<85$ & $85-90$ & 0.6057 (NS) \\
\hline Tachycardia (HR >100/min) & $4(21)$ & $15(22)$ & 0.9882 (NS) \\
\hline Bradycardia $(\mathrm{HR}<60 / \mathrm{min})$ & $1(6)$ & $5(7)$ & 0.7908 (NS) \\
\hline Fever $\left(\geq 38.0^{\circ} \mathrm{C}\right)$ & $5(24)$ & $22(29)$ & 0.6425 (NS) \\
\hline Amount of blood (Fisher scale) & & & 0.0023 \\
\hline $\mathrm{I} / \mathrm{II}$ & $4(19)$ & $2(2.5)$ & \\
\hline III & $9(43)$ & $22(27.5)$ & \\
\hline IV & $8(38)$ & $59(74)$ & \\
\hline WFNS grade & & & $<0.0001$ \\
\hline IV & $15(71)$ & $11(14)$ & \\
\hline $\mathrm{V}$ & $6(29)$ & $69(86)$ & \\
\hline
\end{tabular}


Table 2 Results of multivariate logistic regression

\begin{tabular}{|c|c|c|c|c|}
\hline Variable included in model & Coefficient & SE & OR & p Value \\
\hline WFNS score & 2.80454 & 0.73042 & 16.5195 & 0.0001 \\
\hline Age tertile & 1.58949 & 0.5696 & 4.9013 & 0.0053 \\
\hline Fisher scale & 1.43876 & 0.5135 & 4.2155 & 0.0051 \\
\hline Leucocytosis & 1.81886 & 0.77252 & 6.1649 & 0.0186 \\
\hline \multicolumn{5}{|l|}{ Overall model fit } \\
\hline Null model -2log likelihood & & & & 103.26 \\
\hline Full model -2log likelihood & & & & 55.89 \\
\hline$\chi^{2}$ & & & & 47.371 \\
\hline Degrees of freedom & & & & 4 \\
\hline$p$ Value & & & & $<0.0001$ \\
\hline \multicolumn{5}{|l|}{ Hosmer and Lemeshow test } \\
\hline$\chi^{2}$ & & & & 3.0342 \\
\hline Degrees of freedom & & & & \\
\hline $\mathrm{p}$ Value & & & & 0.9322 \\
\hline
\end{tabular}

\section{Proposed prognostic scale}

On the basis of the analysis, a prognostic model was designed (table 3). The total score is the sum of the points of the various characteristics. WFNS grade, which was strongly associated with the outcome, was given the most weight on the scale. Other factors had lower predictive ability and adjudged to score each of them lower then WFNS grade IV. As the other predictors had relatively the same association with the outcome, they were weighted relatively similarly. The proposed scale had nine theoretical grades, which were collapsed to four grades (risk groups) for simplification. Grades were compressed when differences in poor outcomes were minimal or absent (table 4). The ROC curve for the prediction of unfavourable outcome performed by the new scale had an area under the curve (AUC) $=0.910$ (excellent accuracy). Figure 1 shows a survival curve during the period of observation-the patients classified in the four risk groups had a different survival experience (log-rank

\section{Table 3 Proposed scale characteristics}

\begin{tabular}{lc}
\hline Component & Score point \\
\hline WFNS grade & \\
IV & 3 \\
V & 5 \\
Age, year & \\
$\leq 50$ & 0 \\
$50-65$ & 1 \\
$>65$ & 2 \\
Fisher grade & 0 \\
I, II & 1 \\
III & 2 \\
IV & \\
Leucocytosis & 0 \\
WCC $<15 \times 10^{9} /$ L & 2 \\
WCC $\geq 15 \times 10^{9} / L$ & $3-11$ \\
Total score & \\
\hline WCC, white cell count; WFNS, World Federation of Neurological \\
Surgeons Grading System for Subarachnoid Hemorrhage.
\end{tabular}

$\chi^{2}=25.11 ; \mathrm{p}<0.0001$ ), with a median survival of 5 days for group IV, 7 days for group III and 11 days for group II.

\section{DISCUSSION}

Owing to the advancement of diagnostics and the treatment of patients with $\mathrm{SAH}$, the prognoses for these patients have improved significantly in recent decades. Nevertheless, current epidemiological data still indicate a high percentage of disability (33\%) and deaths (44\%) among patients with $\mathrm{SAH} .{ }^{12}$ Patients who receive only conservative treatment due to a poor clinical condition are an important population that to a great extent predetermines the data.

Both current research conducted on the group of patients who receive only conservative treatment during a 30-day long monitoring and previously published papers that analyse the patients who are surgically treated indicate that the level of consciousness, patient's age and the amount of bleeding in CT neuroimaging-all of

Table 4 Distribution of 101 patients with poor-grade subarachnoid haemorrhage, according to the new grading scale

\begin{tabular}{llcc}
\hline Score & $\begin{array}{l}\text { Favourable } \\
\text { outcome }\end{array}$ & $\begin{array}{l}\text { Unfavourable } \\
\text { outcome }\end{array}$ & Total \\
\hline 3 & 0 & $0(0 \%)$ & 0 \\
4 & 3 & $1(25 \%)$ & 4 \\
5 & 8 & $0(0 \%)$ & 7 \\
6 & 4 & $6(60 \%)$ & 10 \\
7 & 5 & $9(64 \%)$ & 14 \\
8 & 1 & $25(96 \%)$ & 26 \\
9 & 1 & $21(95 \%)$ & 22 \\
10 & 0 & $8(100 \%)$ & 8 \\
11 & 0 & $10(100 \%)$ & 10 \\
$3-5$ & 11 & $1(9.1 \%)$ & 11 \\
$6-7$ & 9 & $15(62.5 \%)$ & 24 \\
$8-9$ & 2 & $46(95.8 \%)$ & 48 \\
$10-11$ & 0 & $18(100 \%)$ & 18 \\
\hline
\end{tabular}




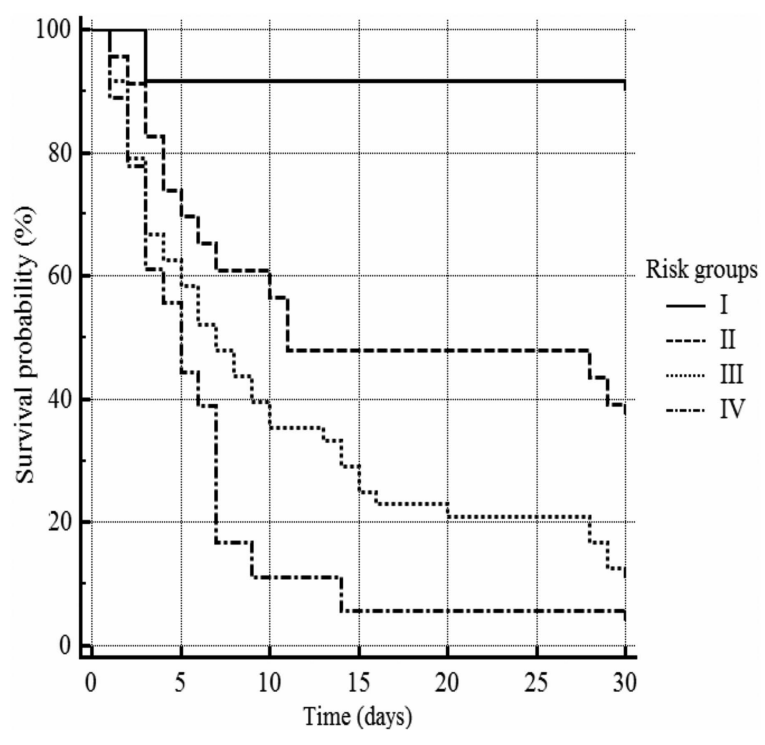

Figure 1 Kaplan-Meier survival curve.

them assessed at the moment of a patient's admission to hospital-are the independent predictive factors after $\mathrm{SAH}$. There is less coherence in various publications as far as other additional factors are concerned, such as cerebral vasospasm (CVS), size and location of the aneurysm, the method according to which the ruptured blood vessel is managed, history of arterial hypertension and some laboratory tests. ${ }^{5}$ The relevance of leucocytosis on the course of the disease after SAH has been reported in surgical cases. However, these studies mainly analysed leucocytosis as a component of systemic inflammatory response syndrome (SIRS) and/or as an aspect of vasospasm pathogenesis. Interestingly, the admission leucocytosis alone correlated independently and extraordinarily highly with outcome in non-surgical cases.

The state of consciousness is a well-documented and prevailing factor describing the later course of the disease. ${ }^{13}$ The first universal tools for assessing the state of consciousness and clinical condition at the same time were Botterel's scale and subsequently its modification, the Hunt and Hess scale. ${ }^{14}{ }^{15}$ However, these models are constructed on the basis of subjective perception, and hence the repeatability of measurement when used. Consequently, the WFNS scale, a tool based on the Glasgow Coma Scale, ${ }^{16}$ was proposed to improve the reliability and significance of grading the level of consciousness. Undoubtedly, the degree of brain tissue damage assessed by WFNS is the most valid predictor of poor outcome in patients with SAH. However, selection of patients for management only on the basis of the neurological condition remains controversial. ${ }^{13}$ Therefore, a more complex evaluation of clinical state by adding other independent prognostic factors is crucial to improve $\mathrm{SAH}$ grading.

Undeniably, the risk of an unfavourable course of the disease increases in proportion to age. Elderly patients frequently experience massive $\mathrm{SAH}$ and a dramatically serious clinical condition as early as at the onset of the disease. ${ }^{17}$ However, the independent predictive value seems to depend on further cerebral and systemic complications. With age, as contrasted with younger patients, raised intracranial pressure is considered less significant for secondary damage of the central nervous system while the significance of arterial hypertension/hypotension and cerebral hypoxia increases. ${ }^{18}$ What is more, chronic conditions that precede the onset of the disease and reduce the level of cardiovascular, respiratory and renal efficiency predispose the patient to serious systemic complications that will occur during his or her stay in hospital. ${ }^{19-21}$ A combination of the factors discussed does manifestly contribute to an unfavourable course of the disease among elderly patients, which gives it special significance in the context of the analysed group of patients.

The amount of blood assessed in particular CT scans proves to be related to the development of vascular spasm that leads to vasospasm and secondary ischaemic brain damage, one of common complication and essential for further course of the disease after the survival of primary cerebral haemorrhage. ${ }^{10}$ However, the indirect pathophysiological mechanism which leads to this compilation remains unknown. The amount of SAH caused increased intracranial pressure, an important mechanism for stopping the bleeding from the ruptured blood vessel and preventing the recurrence of haemorrhage, but at the same time it caused decreased cerebral perfusion and secondary formation of brain oedema effects, thus increasing the intracranial pressure. ${ }^{22} 23$ Several tools employed to determine the severity of SAH have been developed on the basis of CT imaging. They include the Fisher scale, which is a widely used tool in research studies. Our study has confirmed the predictive value of the aforementioned scale and indicated grade IV as the worst predictive value. However, different studies suggest that grade IV incorrectly offers the highest predictive value. $^{24}$ This might have resulted from the analysed population ${ }^{25} 26$ or the fact that the clot with a thickness of over $1 \mathrm{~mm}$ has become outdated nowadays.

As previously mentioned, the increased WCC during the acute phase of SAH are a component of SIRS. This process is commonly observed among those patients and depends on the severity of brain damage and the presence of blood breakdown products in the subarachnoid space. ${ }^{27}$ Despite this, the correlation between SIRS and prognosis seems independent from other prognostic factors. ${ }^{28}$ It is also worth noting that surgical stress and anaesthesia may affect the components of the SIRS score, which may lead to overestimation. ${ }^{29}$ Interestingly, in our study, the connection between other individual SIRS criteria (tachypnoea was not measured) and the course of the disease was ruled out. It seems that leucocytosis is a key component in the process responsible for adverse effects of systemic inflammatory reaction. Previous studies demonstrated the connection between leucocytosis and the development of CVS. ${ }^{7}$ Furthermore, leucocytosis was reported to be associated 
with delayed cerebral ischaemia (DCI) ${ }^{30}$ WCC release a large number of cytokines, including in particular the tumour necrosis factor $(\mathrm{TNF} \alpha)$ and interleukins (IL-1 $\alpha$, IL-1 $\beta$, IL-6, IL-8) which activate and propagate the immune response. These proteins seem to play a key role in the development of CVS, ${ }^{31}{ }^{32}$ which of course can be symptomatic and lead to DCI. Furthermore, in experimental models, leucocytes and platelets adhere to the microvasculature at the cerebral surface immediately after SAH. These early inflammatory and prothrombogenic responses may cause a whole-brain injury immediately after $\mathrm{SAH} .{ }^{33}$

There is a lack of studies that compare elevated WCC and recurrent bleeding from aneurysms. Existing researches have not clearly indicated a relationship between leucocytosis and rebleeding. ${ }^{34}$ However, WCC can play a certain role in this process. Leucocyte infiltration has been well documented in intracranial aneurysms and seems to be associated with primarily aneurysmal wall destruction and rupture. ${ }^{35}$ Thus, leucocytes may induce damage directly to the unstable aneurysm and result in rebleeding.

Our study has confirmed the high morbidity and mortality rates in a non-operative treatment population. However, there are different subgroups of patients with different outcomes. The new scale is a reliable tool that identifies classes of patients with various outcome probabilities, which may contribute to more effective planning of therapeutic management in patients with poor-grade SAH. Another advantage is the fact that the created tool gives the opportunity for earlier use. Nonetheless, further studies are needed to check its performance in a large, prospective analysis in patients suffering poor-grade SAH.

\section{CONCLUSIONS}

The obtained results have allowed us to draw the following conclusions. The natural course of the disease depends on: (1) the degree of consciousness (primary and specific brain damage during SAH); (2) the patient's age (brain plasticity, arteriosclerosis, chronic illness); (3) the size of intracranial bleeding (increasing intracranial pressure, decreased cerebral blood flow, CVS and early and delayed global cerebral oedema). Finally, it also depends on leucocytosis as an indicator of systemic inflammatory activation in response to acute brain injury in the course of SAH. A new predictive scale built on the above parameters seems to reliably predict the outcome in a 30 -day observation period.

Contributors KR, WZ and TT conceived and designed the experiments. SS, $A M$ and AK performed the experiments. SS analysed the data. KR, RR and SS contributed to the writing of the manuscript. The content of the final manuscript was reviewed by all authors.

Funding The study was funded with a scientific grant from the Medical University of Lublin (DS387/10).

Competing interests None declared.

Patient consent Obtained.
Ethics approval The study was approved by the institutional review board of the Medical University of Lublin.

Provenance and peer review Not commissioned; externally peer reviewed.

Data sharing statement No additional data are available.

Open Access This is an Open Access article distributed in accordance with the Creative Commons Attribution Non Commercial (CC BY-NC 4.0) license, which permits others to distribute, remix, adapt, build upon this work noncommercially, and license their derivative works on different terms, provided the original work is properly cited and the use is non-commercial. See: http:// creativecommons.org/licenses/by-nc/4.0/

\section{REFERENCES}

1. Hutchinson PJ, Power DM, Tripathi $P$, et al. Outcome from poor grade aneurysmal subarachnoid haemorrhage-which poor grade subarachnoid hemorrhage patients benefit from aneurysm clipping? Br J Neurosurg 2000;14:105-9.

2. Laidlaw JD, Siu KH. Poor-grade aneurysmal subarachnoid hemorrhage: outcome after treatment with urgent surgery. Neurosurgery 2003;53:1275-80.

3. Gupta SK, Ghanta RK, Chhabra R, et al. Poor-grade subarachnoid hemorrhage: is surgical clipping worthwhile? Neurol India 2011;59:212-17.

4. Connolly ES Jr, Rabinstein AA, Carhuapoma JR, et al. Guidelines for the management of aneurysmal subarachnoid hemorrhage: a guideline for healthcare professionals from the American Heart Association/American Stroke Association. Stroke 2012;43:1711-37.

5. Jaja BN, Cusimano MD, Etminan N, et al. Clinical prediction models for aneurysmal subarachnoid hemorrhage: a systematic review. Neurocrit Care 2013;18:143-53.

6. Drake CG, Hunt WE, Sano K, et al. Report of World Federation of Neurological Surgeons Committee on a Universal Subarachnoid Hemorrhage Grading Scale. J Neurosurg 1988;68:985-6.

7. McGirt MJ, Mavropoulos JC, McGirt LY, et al. Leukocytosis as an independent risk factor for cerebral vasospasm following aneurysmal subarachnoid hemorrhage. J Neurosurg 2003;98:1222-6.

8. Van Assche E, De Deyne C, Schneider I, et al. Early leukocytosis occurring after subarachnoid hemorrhage could be used as a predictor of later cerebral vasospasm: A-349. Eur J Anaesthesiol 2006;23:26.

9. Schlenk F, Vajkoczy P, Sarrafzadeh A. Inpatient hyperglycemia following aneurysmal subarachnoid hemorrhage: relation to cerebral metabolism and outcome. Neurocrit Care 2009;11:56-63.

10. Fisher CM, Kistler JP, Davis JM. Relation of cerebral vasospasm to subarachnoid hemorrhage visualized by computerized tomographic scanning. Neurosurgery 1980;6:1-9.

11. Helbok R, Ko SB, Schmidt JM, et al. Global cerebral edema and brain metabolism after subarachnoid hemorrhage. Stroke 2011;42:1534-9.

12. Nieuwkamp DJ, Setz LE, Algra A, et al. Changes in case fatality of aneurysmal subarachnoid haemorrhage over time, according to age, sex, and region: a meta-analysis. Lancet Neurol 2009;8:635-42.

13. Lagares A, Gómez PA, Lobato RD, et al. Prognostic factors on hospital admission after spontaneous subarachnoid haemorrhage. Acta Neurochir (Wien) 2001;143:665-72.

14. Botterel EH, Lougheed WM, Scott JW, et al. Hypothermia, and interruption of carotid, or carotid and vertebral circulation, in the surgical management of intracranial aneurysms. J Neurosurg 1956;13:1-42.

15. Hunt WE, Hess RM. Surgical risk as related to time of intervention in the repair of intracranial aneurysms. J Neurosurg 1968;28:14-20.

16. Teasdale G, Jennett B. Assessment of coma and impaired consciousness. A practical scale. Lancet 1974;2:81-4.

17. Lanzino G, Kassell NF, Germanson TP, et al. Age and outcome after aneurysmal subarachnoid hemorrhage: why do older patients fare worse? J Neurosurg 1996;85:410-18.

18. Ryttlefors M, Howells T, Ronne-Engström E, et al. Neurointensive care is justified in elderly patients with severe subarachnoid hemorrhage-an outcome and secondary insults study. Acta Neurochir (Wien) 2010;152:241-9.

19. Sprung J, Gajic O, Warner DO. Review article: age related alterations in respiratory function-anesthetic considerations. Can J Anaesth 2006;53:1244-57.

20. Beck LH. Changes in renal function with aging. Clin Geriatr Med 1998;14:199-209.

21. Cheitlin MD. Cardiovascular physiology_changes with aging. Am J Geriatr Cardiol 2003;12:9-13. 
22. McCormick PW, McCormick J, Zabramski JM, et al. Hemodynamics of subarachnoid hemorrhage arrest. J Neurosurg 1994;80:710-15.

23. Claassen J, Carhuapoma JR, Kreiter KT, et al. Global cerebral edema after subarachnoid hemorrhage: frequency, predictors, and impact on outcome. Stroke 2002;33:1225-32.

24. Frontera JA, Claassen J, Schmidt JM, et al. Prediction of symptomatic vasospasm after subarachnoid hemorrhage: the modified fisher scale. Neurosurgery 2006;59:21-7.

25. Le Roux PD, Elliott JP, Newell DW, et al. Predicting outcome in poor-grade patients with subarachnoid hemorrhage: a retrospective review of 159 aggressively managed cases. $J$ Neurosurg 1996;85:39-49.

26. Shimoda M, Oda S, Shibata M, et al. Results of early surgical evacuation of packed intraventricular hemorrhage from aneurysm rupture in patients with poor-grade subarachnoid hemorrhage. J Neurosurg 1999;91:408-14.

27. Yoshimoto Y, Tanaka Y, Hoya K. Acute systemic inflammatory response syndrome in subarachnoid hemorrhage. Stroke 2001;32:1989-93.

28. Tam AK, llodigwe D, Mocco J, et al. Impact of systemic inflammatory response syndrome on vasospasm, cerebral infarction, and outcome after subarachnoid hemorrhage: exploratory analysis of CONSCIOUS-1 database. Neurocrit Care 2010;13:182-9.
29. Desborough JP. The stress response to trauma and surgery. Br J Anaesth 2000;85:109-17.

30. Kasius KM, Frijns CJ, Algra A, et al. Association of platelet and leukocyte counts with delayed cerebral ischemia in aneurysmal subarachnoid hemorrhage. Cerebrovasc Dis 2010;29:576-83.

31. Fassbender K, Hodapp B, Rossol S, et al. Inflammatory cytokines in subarachnoid haemorrhage: association with abnormal blood flow velocities in basal cerebral arteries. J Neurol Neurosurg Psychiatry 2001;70:534-7.

32. Takizawa T, Tada T, Kitazawa K, et al. Inflammatory cytokine cascade released by leukocytes in cerebrospinal fluid after subarachnoid hemorrhage. Neurol Res 2001;23:724-30.

33. Ishikawa M, Kusaka G, Yamaguchi N, et al. Platelet and leukocyte adhesion in the microvasculature at the cerebral surface immediately after subarachnoid hemorrhage. Neurosurgery 2009;64:546-53.

34. Cong W, Zhongxin Z, Tiangui L, et al. Risk factors for rebleeding of aneurysmal subarachnoid hemorrhage based on the analysis of on-admission information. Turk Neurosurg 2012;22:675-81.

35. Frösen J, Piippo A, Paetau A, et al. Remodeling of saccular cerebral artery aneurysm wall is associated with rupture: histological analysis of 24 unruptured and 42 ruptured cases. Stroke 2004;35:2287-93. 